\title{
Green Synthesis and Antibacterial Activity of Pyrido[2,3-e]1,3,4-Oxadiazine Derivatives
}

\author{
Fayez Mohamed Eissa ${ }^{1,}$, , Mohamed Selim², Ahmed Sayed ${ }^{2}$, Mohamed Sharkawi² $^{2}$ \\ ${ }^{1}$ Chemistry Department, Faculty of Science, Aswan University, Aswan, Egypt \\ ${ }^{2}$ Chemistry Department, Faculty of Science, Al-Azhar University, Cairo, Egypt
}

\section{Email address:}

fayezeissa@gmail.com (F. M. Eissa)

"Corresponding author

\section{To cite this article:}

Fayez Mohamed Eissa, Mohamed Selim, Ahmed Sayed, Mohamed Sharkawi. Green Synthesis and Antibacterial Activity of Pyrido[2,3e]1,3,4-Oxadiazine Derivatives. American Journal of Heterocyclic Chemistry. Vol. 5, No. 3, 2019, pp. 71-75. doi: 10.11648/j.ajhc.20190503.13

Received: June 22, 2019; Accepted: July 13, 2019; Published: August 22, 2019

\begin{abstract}
Pyrido[2,3-e]1,3,4-Oxadiazines heterocyclic ring system and its derivatives were efficiently synthesized via benign and sustainable methodology using Grinding Chemistry as a recognized solvent free, less hazard, and low risk pathway. The course of the reaction was completed by refluxing using ecofriendly and renewable source of initiation energy via means of parabolic solar reflectors. The implemented parabolic solar reflectors employed the direct sunlight as a clean and sustainable source of activation energy to activate the required chemical reaction. The green context of the procedure was evaluated and discussed by comparing its benign components with the non-green components of the conventional one. The green components of the green procedures were found virtually preferred over the conventional one with its simplicity, time efficiency, atom economy, safety, sustainability, low cost, yield optimization, excellent conversion of the reactants and ease of applicability. The biological activity of the prepared compounds was evaluated and discussed against some bacterial strains naming, (Escherichia Coli, Pseudomonas, Serratia and Bacillus Cereus), and was found to display promising anti-bacterial results that encourage future pharmaceutical studies to quantify its potency. All the synthesized compounds were purified using glass column chromatography packed with silica and their structures were identified and confirmed by elemental analysis and Mass spectra, ${ }^{1} \mathrm{HNMR},{ }^{13} \mathrm{CNMR}$ spectral data.
\end{abstract}

Keywords: Green Synthesis, Antibacterial, Pyrido[2,3-e]1,3,4-Oxadiazines, Solar Reflectors, Heterocycles, Grinding Chemistry

\section{Introduction}

Introducing new heterocycles that can react with biological organisms as drug leads has shaped a growing requisition for competent synthetic methodologies leading to diverse and efficient "therapeutic-like" structures [1-3].

1, 3, 4-Oxadiazines are important heterocyclic moieties with its great antibacterial and antifungal activities [4-7]. They have a promising location in the design and production of proficient biologically active materials and still continue to draw attention due to their broad spectrum and diversity [8]. Moreover, their derivatives showed important roles in valued biological activities [9-11], antinflammation [12, 13], against malaria [14], antituberculosis [15-21], and anticancer [22].

Heterocycles were commonly established through traditional procedures and it made their production environmentally non proficient process [23]. Currently, simple, benign and sustainable synthetic tactics for Pyrido[2,3-e]1,3,4-Oxadiazines derivatives was carried out. Both grinding of starting material and refluxing via means of parabolic solar reflector was the feasible green, simple, and rapid method for the synthesis instead of the hazardous traditional procedures [24-26]. The assembled benign method introduced yield optimization with excellent reaction conversion. Moreover, refluxing with parabolic solar reflectors presented energy efficient, economically constructive, and sustainable source of activation to establish such valuable organic compounds. 


\section{Results and Discussion}

\subsection{Synthesis}

Pyrido[2,3-e]1,3,4-Oxadiazines derivatives, 3a-g, were prepared through benign and sustainable way employing grinding and refluxing via means of parabolic solar reflector as renewable and ecofriendly sources of energy.

Initially, 3-bromopyridin-2-ol, 2, was established by bromination of 2-pyridinone through atom efficient and green process in which corrosive bromine was avoided, water was the only byproduct and $\mathrm{HBr}-\mathrm{H}_{2} \mathrm{O}_{2}$ mixture and bromidebromate couple offer in situ oxidation of $\mathrm{Br}^{-}$to molecular bromine.

Furthermore, Pyrido[2,3-e]1,3,4-Oxadiazines derivatives, 3a-g, were obtained via grinding 3-bromopyridin-2-ol, 1, with hydrazonic acid derivatives, $2 \mathrm{a}-\mathrm{g}$, then refluxing by solar reflectors in ethanol containing sodium bicarbonate as basic catalyst, Figure 1.

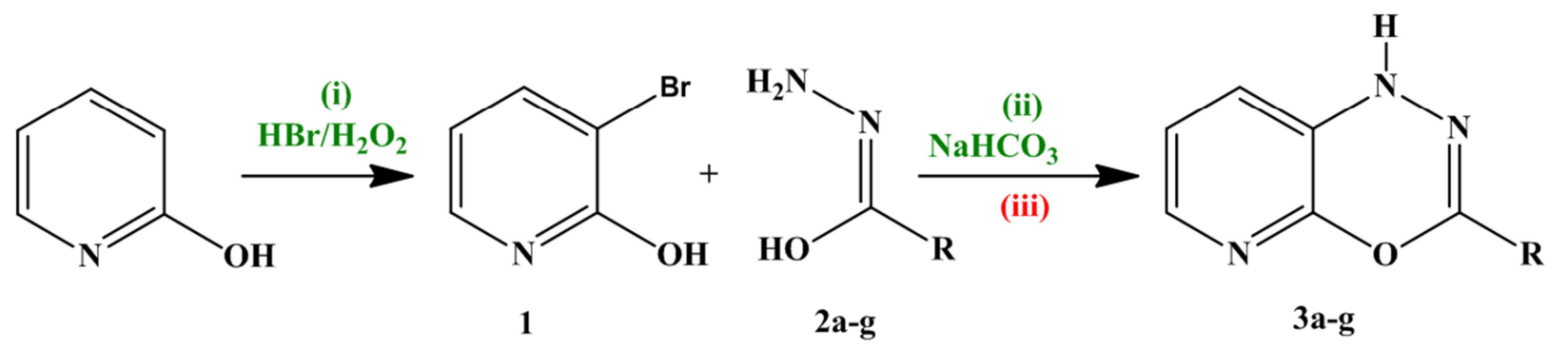

\begin{tabular}{|c|c|c|c|c|c|c|}
\hline $\begin{array}{l}\text { Green Yield: } 93 \% \text {, } \\
\text { Non Green Y: 71\%, }\end{array}$ & $\begin{array}{l}92 \%, \\
69 \%,\end{array}$ & $\begin{array}{l}85 \%, \\
63 \%,\end{array}$ & $\begin{array}{c}79 \%, \\
61 \%\end{array}$ & $\begin{array}{l}89 \%, \\
70 \%,\end{array}$ & $\begin{array}{l}78 \%, \\
67 \%,\end{array}$ & $\begin{array}{l}90 \% \\
70 \%\end{array}$ \\
\hline
\end{tabular}

Figure 1. (i) Refluxing via means of solar reflector(ii) Green: Grinding 10 minutes then refluxing via solar reflectors, $1 \mathrm{hr}$ in EtOH/NaHCO (iii) Non green: refluxing via heating in dark, 5 hrs in $\mathrm{EtOH} / \mathrm{NaHCO}_{3}$.

The reaction activation energy was supplied through green chemistry and sustainable methods via initiation through grinding the reacting materials at room temperature, then, propagation through refluxing by means of parabolic solar reflector. Grinding at room temperature supplied the reacting materials efficient contact by friction and gentle activation energy to initiate. Moreover, extra refluxing in ethanol via concentrated solar energy, parabolic solar reflector, provided the reaction enough propagation. The optimized reaction yields and the decreased reaction time claimed that the reaction proceeded via both ionic and free radical mechanisms.

According to the suggested mechanism, the nitrogen, with its lone pair, would attack the positively polarized carbon after complete departure of the bromide ion resulting in a unimolecular Nucleophilic Substitution, $\left(\mathrm{SN}_{1}\right)$. This claim was reinforced by facts that bromide is a good leaving group and the resulted electron deficient carbocation would be stabilized through charge delocalization via pyridine ring, Figure 2. Moreover, the oxygen, with its lone pair, would attack the electron deficient carbon of the carbonyl. Concurrently, the hydroxyl would leave it resulting in a bimolecular Nucleophilic Substitution $\left(\mathrm{SN}_{2}\right)$. Consequently, the ring closure would be at complete departure of the hydroxide ion Figure 2.

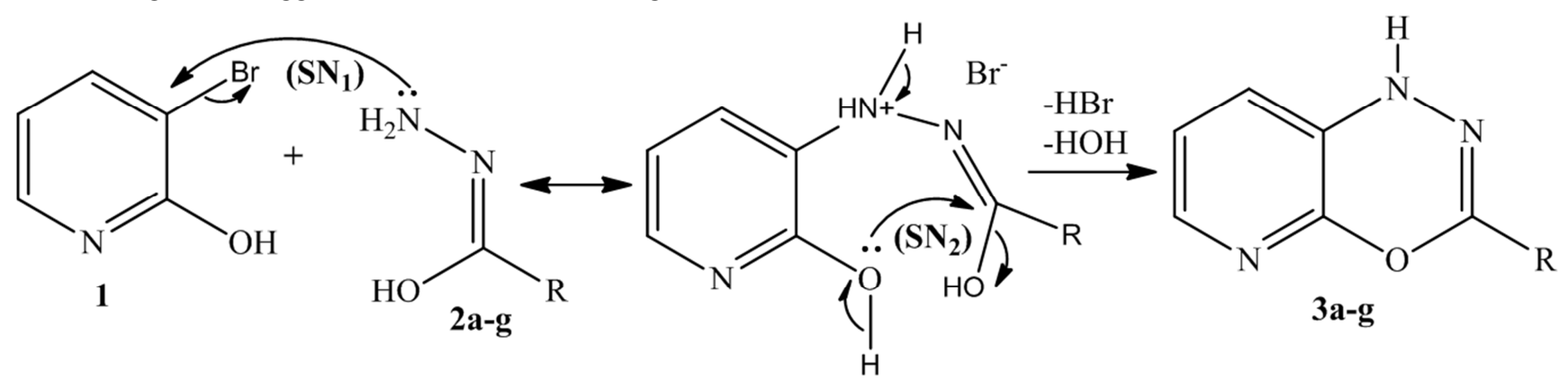

Figure 2. Simple suggestion of the ionic mechanism of preparation of compounds $3 a-g$.

The free radical mechanism would be initiated via UV irradiation by means of parabolic solar reflector that was engaged in the green procedure. The polarized bonds would be cleaved homiletically, resulting free radicals that continue propagating the synthesis according to free radical mechanism sequences. 
Conventionally, the above synthesis could be done via refluxing the reactants mixture in ethanol containing sodium bicarbonate as basic catalyst. The non-green components of this procedure were obvious, longer reaction time with much heating energy, low yield with low atom economy, and nonsustainable way that pointed out the superiority of the green procedure.

The benign and sustainable context of the green procedure is clear enough and its superiority over the conventional one is largely recognized and suitable for planning facile, renewable, energy proficient, excellent conversion with optimal yield, and high atom economy chemistry practices.

\subsection{Antibacterial Activity}

The antibacterial activity was achieved by testing the Pyrido[2,3-e]1,3,4-Oxadiazines derivatives, 3a-g against Escherichia Coli, Pseudomonas, Serratia and Bacillus Cereus, Table 1. Almost, all tested compounds exhibited encouraging results that could be mainly accredited to the
Pyrido[2,3-e]1,3,4-Oxadiazine moiety with its high antibacterial activity. Obviously, Chlorine atoms in derivatives $3 \mathrm{c}$, 3d had largely increased activity against all the bacterial strains due to the suggested efficacy against bacterial growth of the chlorine atoms attached to heterocycles [27]. Also, the compound with methoxyl group $3 \mathrm{f}$ revealed high activity that could be attributed to the lipophilic character of such compounds and the increased growth inhibition of the bacterial strains. Evidently, compound $3 \mathrm{~g}$ showed high antibacterial activity due to the extra influence by the pyridine side residue beside the Pyrido[2,3-e]1,3,4-Oxadiazine moiety. Mostly, presence of side chain residues increased the antibacterial activity since the branching increase the intractability with bacteria, hence, directly affected the bacterial growth. Remarkably, all the compounds (3a-g) showed promising antibacterial activity compared to the reference and this indicate the importance of the Pyrido[2,3-e]1,3,4-Oxadiazine ring system.

Table 1. Antibacterial activity of the test compounds $3 a-g$.

\begin{tabular}{llll}
\hline \multirow{2}{*}{ Comp. } & \multicolumn{2}{l}{ Inhibition Zone Diameter IZD(activity) } \\
\cline { 2 - 4 } & Escherichia Coli & Pseudomonas & Serattia \\
\hline $3 \mathrm{a}$ & $14 \mathrm{~mm}(+)$ & $16 \mathrm{~mm}(++)$ & $17 \mathrm{~mm}(++)$ \\
$3 \mathrm{~b}$ & $13 \mathrm{~mm}(+)$ & $20 \mathrm{~mm}(+++)$ & $21 \mathrm{~mm}(+++)$ \\
$3 \mathrm{c}$ & $18 \mathrm{~mm}(++)$ & $19 \mathrm{~mm}(++)$ & $21 \mathrm{~mm}(+++)$ \\
$3 \mathrm{~d}$ & $19 \mathrm{~mm}(++)$ & $20 \mathrm{~mm}(+++)$ & $19 \mathrm{~mm}(++)$ \\
$3 \mathrm{e}$ & $13 \mathrm{~mm}(+)$ & $18 \mathrm{~mm}(++)$ & $15 \mathrm{~mm}(++)$ \\
$3 \mathrm{f}$ & $17 \mathrm{~mm}(++)$ & $19 \mathrm{~mm}(++)$ & $21 \mathrm{~mm}(+++)$ \\
$3 \mathrm{~g}$ & $19 \mathrm{~mm}(++)$ & $20 \mathrm{~mm}(+++)$ & $22 \mathrm{~mm}(+++)$ \\
Control & $05 \mathrm{~mm}(-)$ & $05 \mathrm{~mm}(-)$ & $05 \mathrm{~mm}(-)$ \\
Ampicillin & $21 \mathrm{~mm}(+++)$ & $22 \mathrm{~mm}(+++)$ & $23 \mathrm{~mm}(+++)$ \\
\hline
\end{tabular}

\section{Experimental}

\subsection{General}

All reagents were purchased from dealers of Merck Serono Egypt, in Cairo, and were used without further purification. Solvents were of analytical grade. Melting points were determined in open glass capillaries on a Gallenkamp melting point apparatus and were uncorrected. Elemental analysis was performed on a Thermo Finnigan Flash EA 1112 Element Analyzer (Italy). ${ }^{1} \mathrm{HNMR}$ and ${ }^{13} \mathrm{CNMR}$ spectra were recorded on a Varian Mercury $300 \mathrm{MHz}$ spectrometer using Tetramethylsilane as an internal standard and DMSO- $d 6$ as solvent. Mass spectra were recorded at $70 \mathrm{eV}$ on a Shimadzu GCMS-QP1000EX using an inlet type injector. All reactions were monitored and the products were confirmed by TLC(silica gel, aluminum sheets 60 F254, Merck). All Figures were regenerated via Chem. Draw Ultra 12.0.2.1076.

\subsection{Synthesis}

3-bromopyridin-2-ol, (1).

Pridin-2-one $(0.01 \mathrm{~mol})$ in ethanol $(10 \mathrm{ml})$ was refluxed via means of parabolic solar reflector. The aqueous solution of $\mathrm{HBr}(33 \%)(5.2 \mathrm{ml})$ and hydrogen peroxide $\left(\mathrm{H}_{2} \mathrm{O}_{2}, 30 \%\right)(7 \mathrm{ml})$ were added from a dropping funnel sequentially to this refluxing solution of pridin-2-one. The red color disappeared within 15 minutes. This indicated the bromination of pridin2-one, then, the solution was allowed to cool down. During this the precipitate due to 3-bromopriding-2-ol, 1, was separated out. The precipitate was filtered, recrystallized from ethanol and dried. The structure of the resulting compound was confirmed by elemental analysis and spectroscopy.

M.P.:111-113 Co; M. F.: C5H4BrNO, M. Wt.: 174.00, m/z: 172.95, Elemental analysis:(Calculated \%) C, 34.51; H, 2.32; Br, 45.92; N, 8.05; O, 9.20;(Found \%) C, 34.48; H, 2.35; Br, 45.90; N, 8.09; ${ }^{1} \mathrm{HNMR}: 6.34(\mathrm{t}, 1 \mathrm{H}-\mathrm{Ar}), 7.51(\mathrm{~d}, 1 \mathrm{H}-\mathrm{Ar}$.$) ,$ 7.93(d, 1H-Ar), 11.01(s, 1H-OH); ${ }^{13} \mathrm{CNMR}: 105.15(\mathrm{CH}-\mathrm{C} 5)$, 117.51(C-C3), 140.15(CH-C6), 144.19(CH-C4), 161.91(CC2).

Pyrido[2,3-e]1,3,4-Oxadiazine derivatives(3a-g)

The starting compounds 1 and hydrazonic acid derivatives(2a-g), in equimolar ratios, (0.01 mole), and three drops of aqueous $\mathrm{NaHCO}_{3}(20 \%)$, were grinded for 10 minutes, by means of agate mortar and pestle at room temperature. The resulted reddish paste was refluxed in 20 $\mathrm{mL}$ ethanol 95\%, via means of solar reflector, with continuous stirring to retain excellent conversion and optimum yield. The solvent was recycled by evaporation. The remainder crystals of Pyrido[2,3-e]1,3,4-Oxadiazines, (3a-g), were extra purified using column chromatography 
eluting with suitable hexane-methylene chloride developer matrix(80/20). All the synthesized compounds were characterized and confirmed via elemental analysis and Mass, ${ }^{1} \mathrm{HNMR},{ }^{13} \mathrm{CNMR}$ spectroscopy.

3-methyl-1H-pyrido[3, 2-e][1, 3, 4] oxadiazine(3a).

M. P.: 165-167 C ; M. F.: $\mathrm{C}_{7} \mathrm{H}_{7} \mathrm{~N}_{3} \mathrm{O}$, M. Wt.: $149.15, \mathrm{~m} / \mathrm{z}$ : 149.06, Elemental analysis:(Calculated \%) C, 56.37; H, 4.73; N, 28.17; O, 10.73;(Found \%) C, 56.40; H, 4.75; N 28.11; ${ }^{1} \mathrm{HNMR}: 1.18(\mathrm{~s}, 3 \mathrm{H}-\mathrm{CH} 3), 6.12(\mathrm{~s}, 1 \mathrm{H}-\mathrm{NH}),. 6.7\left(\mathrm{t}, 1 \mathrm{H}-\mathrm{C}_{7}\right)$, 7.1(d, $\left.1 \mathrm{H}_{-} \mathrm{C}_{8}\right), \quad 7.5\left(\mathrm{~s}, \quad 1 \mathrm{H}-\mathrm{C}_{6}\right)$; ${ }^{13} \mathrm{CNMR}: 21.5(-\mathrm{CH} 3)$, $112.3\left(\mathrm{CH}-\mathrm{C}_{7}\right), 117.5\left(\mathrm{CH}-\mathrm{C}_{6}\right), 127.1\left(\mathrm{CH}-\mathrm{C}_{8}\right), 131.5\left(\mathrm{C}-\mathrm{C}_{5}\right)$, $142.9\left(\mathrm{C}-\mathrm{C}_{2}\right), 151.1\left(\mathrm{C}-\mathrm{C}_{10}\right)$.

3-phenyl-1H-pyrido[3, 2-e][1, 3, 4]oxadiazine(3b).

M. P.: 172-173 C ; M. F.: $\mathrm{C}_{12} \mathrm{H}_{9} \mathrm{~N}_{3} \mathrm{O}, \mathrm{M}$. Wt.: $211.22, \mathrm{~m} / \mathrm{z}$ : 211.22, Elemental analysis:(Calculated \%) C, 68.24; H, 4.29; N, 19.89; O, 7.57;(Found \%) C, 68.21; H, 4.31; N, 19.87; ${ }^{1} \mathrm{HNMR}: \quad 5.9(\mathrm{~s}, 1 \mathrm{H}-\mathrm{NH}),. 6.4\left(\mathrm{t}, 1 \mathrm{H}-\mathrm{C}_{12}\right), 6.7\left(\mathrm{~d}, 1 \mathrm{H}-\mathrm{C}_{13}\right)$, 7.1.(s, $\left.1 \mathrm{H}-\mathrm{C}_{11}\right), 7.51\left(\mathrm{~d}, 1 \mathrm{H}-\mathrm{C}_{1,2,6}\right) 7.9\left(\mathrm{~s}, 1 \mathrm{H}-\mathrm{C}_{3,5}\right) ;{ }^{13} \mathrm{CNMR}$ : $110.2\left(\mathrm{CH}-\mathrm{C}_{12}\right), 116.7\left(\mathrm{CH}-\mathrm{C}_{11}\right), 122.3\left(\mathrm{CH}-\mathrm{C}_{3,5}\right), 126.5(\mathrm{CH}-$ $\left.\mathrm{C}_{2,6}\right), 128.3\left(\mathrm{C}-\mathrm{C}_{4}\right), 129.5\left(\mathrm{C}-\mathrm{C}_{10}\right), 131.8\left(\mathrm{CH}-\mathrm{C}_{1}\right) .143 .1\left(\mathrm{C}-\mathrm{C}_{7}\right)$ $149,2\left(\mathrm{C}-\mathrm{C}_{15}\right)$.

3-(4-chlorophenyl)-1H-pyrido[3, $\quad 2$-e $][1$, 3 , 4]oxadiazine (3c).

M. P.: 177-178 C ; M. F.: $\mathrm{C}_{12} \mathrm{H}_{8} \mathrm{ClN}_{3} \mathrm{O}$, M. Wt.: 245.66, $\mathrm{m} / \mathrm{z}$ : 245.04, Elemental analysis:(Calculated \%) C, 58.67; H, 3.28; Cl, 14.43; N, 17.10; O, 6.51;(Found \%) C, 58.64; H, $3.31 ; \mathrm{Cl}, 14.41 ; \mathrm{N}, 17.07 ;{ }^{1} \mathrm{HNMR}: 5.8(\mathrm{~s}, 1 \mathrm{H}-\mathrm{NH}), 6.4(\mathrm{~d}$, $\left.1 \mathrm{H}-\mathrm{C}_{13}\right), 6.8\left(\mathrm{t}, 1 \mathrm{H}-\mathrm{C}_{12}\right), 7.2\left(\mathrm{~d}, 1 \mathrm{H}-\mathrm{C}_{11}\right), 7.6\left(\mathrm{~d}, 1 \mathrm{H}-\mathrm{C}_{2,6}\right), 7.8(\mathrm{~s}$, $\left.1 \mathrm{H}-\mathrm{C}_{3,5}\right)$; ${ }^{13} \mathrm{CNMR}: \quad 115.2\left(\mathrm{CH}-\mathrm{C}_{12}\right), \quad 121.7\left(\mathrm{CH}-\mathrm{C}_{11}\right)$, $124.1\left(\mathrm{CH}-\mathrm{C}_{1}\right), \quad 128.3\left(\mathrm{CH}-\mathrm{C}_{4}\right), \quad 129.5\left(\mathrm{CH}-\mathrm{C}_{2,3,5,6}\right), \quad 130.1(\mathrm{C}-$ $\left.\mathrm{C}_{13}\right), 137.7\left(\mathrm{C}-\mathrm{C}_{10}\right), 144.4\left(\mathrm{C}-\mathrm{C}_{7}\right), 146,2\left(\mathrm{C}-\mathrm{C}_{15}\right)$.

3-(2, 4-dichlorophenyl)-1H-pyrido[3, 2-e][1, 3, 4]oxadiazine (3d).

M. P.: 180-182 C'; M. F.: $\mathrm{C}_{12} \mathrm{H}_{7} \mathrm{Cl}_{2} \mathrm{~N}_{3} \mathrm{O}$, M. Wt.: 280.11, $\mathrm{m} / \mathrm{z}$ : 279.1, Elemental analysis:(Calculated \%) C, 51.45; H, 2.52; Cl, 25.31; N, 15.00; O, 5.71;(Found \%) C, 51.43; H 2.57; N, 14.97; ${ }^{1} \mathrm{HNMR}: 5.9(\mathrm{~s}, 1 \mathrm{H}-\mathrm{NH}),. 6.82\left(\mathrm{t}, 1 \mathrm{H}-\mathrm{C}_{12}\right)$, 7.21(s, $\left.1 \mathrm{H}-\mathrm{C}_{13}\right), 7.40\left(\mathrm{~s}, 1 \mathrm{H}-\mathrm{C}_{6}\right), 7.56\left(\mathrm{~d}, 1 \mathrm{H}-\mathrm{C}_{11}\right), 7.70(1 \mathrm{H}-$ $\left.\mathrm{C}_{2}\right), 7.80\left(1 \mathrm{H}-\mathrm{C}_{5}\right) ;{ }^{13} \mathrm{CNMR}: 112.2\left(\mathrm{CH}-\mathrm{C}_{12}\right), 120.7\left(\mathrm{CH}-\mathrm{C}_{11}\right)$, $125.1\left(\mathrm{CH}-\mathrm{C}_{6}\right), \quad 128.3\left(\mathrm{C}-\mathrm{C}_{1}\right), \quad 129.5\left(\mathrm{C}-\mathrm{C}_{4}\right), \quad 128.8\left(\mathrm{CH}-\mathrm{C}_{5}\right)$, $129.9\left(\mathrm{CH}-\mathrm{C}_{2}\right), \quad 132.5\left(\mathrm{CH}-\mathrm{C}_{13}\right), \quad 135.7\left(\mathrm{C}-\mathrm{C}_{3}\right), \quad 137.4\left(\mathrm{C}-\mathrm{C}_{10}\right)$, 142.2(C-C $), 149.3\left(\mathrm{C}-\mathrm{C}_{15}\right)$.

3-(p-tolyl)-1H-pyrido[3, 2-e][1, 3, 4]oxadiazine (3e).

M. P.: 192-193 C' ; M. F.: $\mathrm{C}_{13} \mathrm{H}_{11} \mathrm{~N}_{3} \mathrm{O}$, M. Wt.: 225.25, m/z: 225.09, Elemental analysis:(Calculated \%) C, 69.32; H, 4.92; N, 18.66; O, 7.10;(Found \%) C, 69.30; H, 4.96; N, 18.63; ${ }^{1} \mathrm{HNMR}: 2.32\left(\mathrm{~s}, 3 \mathrm{H}-\mathrm{CH}_{3}\right.$.), 5.92(s, 1H-NH), 6.53(d, $\left.1 \mathrm{H}-\mathrm{C}_{14}\right)$, 6.70(t, $\left.1 \mathrm{H}-\mathrm{C}_{13}\right), 7.22\left(\mathrm{~d}, 1 \mathrm{H}-\mathrm{C}_{12}\right), 7.51\left(\mathrm{~d}, 1 \mathrm{H}-{ }_{\mathrm{C} 3,7}\right), 7.96(\mathrm{~d}, 1 \mathrm{H}-$ $\left.\mathrm{C}_{4,6}\right)$; ${ }^{13} \mathrm{CNMR}: 21.5\left(\mathrm{CH}_{3}\right), 111.7\left(\mathrm{CH}-\mathrm{C}_{13}\right), 116.2\left(\mathrm{CH}-\mathrm{C}_{12}\right)$, $120.3\left(\mathrm{C}-\mathrm{C}_{5}\right), 126.3\left(\mathrm{C}-\mathrm{C}_{14}\right), 129.5\left(\mathrm{CH}-\mathrm{C}_{3,4,6,7}\right), 130.2\left(\mathrm{C}-\mathrm{C}_{2}\right)$, $139.1\left(\mathrm{C}-\mathrm{C}_{11}\right), 141,8\left(\mathrm{C}-\mathrm{C}_{8}\right), 148.6\left(\mathrm{C}-\mathrm{C}_{16}\right)$.

3-(4-methoxyphenyl)-1H-pyrido[3, 2-e][1, 3 ,

4]oxadiazine(3f).

M. P.: 180-181 C C $^{\mathrm{O}}$ M. F.: $\mathrm{C}_{13} \mathrm{H}_{11} \mathrm{~N}_{3} \mathrm{O}$, M. Wt.: 241.25, m/z: 241.09, Elemental analysis:(Calculated \%) C, 64.72; H, 4.60; N, 17.42; O, 13.26;(Found \%) C, 64.71; H, 4.63; N, 17.39; ${ }^{1}$ HNMR: 3.75(s, 3H-CH 3 .), 5.90(s, $\left.1 \mathrm{H}-\mathrm{NH}.\right), 6.20$ (s, $\left.1 \mathrm{H}-\mathrm{C}_{14}\right)$, 6.73(t, $\left.1 \mathrm{H}-\mathrm{C}_{15}\right), 7.10\left(\mathrm{~s}, 1 \mathrm{H}-\mathrm{C}_{13}\right), 7.72\left(\mathrm{~d}, 1 \mathrm{H}-\mathrm{C}_{4,8}\right), 8,11(\mathrm{~s}$, 1H- $\left.\mathrm{C}_{5,7}\right)$; ${ }^{13} \mathrm{CNMR}: \quad 57.5\left(\mathrm{CH}_{3}\right), 111.8\left(\mathrm{CH}-\mathrm{C}_{14}\right), 115.7(\mathrm{CH}-$ $\left.\mathrm{C}_{4,8}\right), 119.8\left(\mathrm{CH}-\mathrm{C}_{13}\right), 122.6\left(\mathrm{C}-\mathrm{C}_{6}\right), 126.1\left(\mathrm{CH}-\mathrm{C}_{15}\right), 129.2(\mathrm{C}-$ $\left.\mathrm{C}_{5,7}\right), 129.9\left(\mathrm{C}-\mathrm{C}_{12}\right), 142.4\left(\mathrm{C}-\mathrm{C}_{9}\right), 146,2\left(\mathrm{C}-\mathrm{C}_{17}\right), 162.6\left(\mathrm{C}-\mathrm{C}_{3}\right)$. 3-(pyridin-4-yl)-1H-pyrido[3, 2-e][1, 3, 4]oxadiazine(3g).

M. P.: 197-199 C ; M. F.: $\mathrm{C}_{11} \mathrm{H}_{8} \mathrm{~N}_{4} \mathrm{O}$, M. Wt.: 212.21, m/z: 212.07, Elemental analysis:(Calculated \%) C, 62.26; H, 3.80; N, 26.40; O, 7.54;(Found \%) C, 62.23; H, 3.84; N, 26.38; $\left.{ }^{1} \mathrm{HNMR}: 5.81(\mathrm{~s}, 1 \mathrm{H}-\mathrm{NH}), 1 \mathrm{H}-\mathrm{C}_{8}\right), 6.39\left(\mathrm{t}, 1 \mathrm{H}-\mathrm{C}_{12}\right), 7.23(\mathrm{~d}$, $\left.1 \mathrm{H}-\mathrm{C}_{13}\right), \quad 7.73\left(\mathrm{~d}, \quad 1 \mathrm{H}-\mathrm{C}_{11}\right), \quad 8.11\left(\mathrm{H}-\mathrm{C}_{3,5}\right), \quad 8.70\left(\mathrm{H}-\mathrm{C}_{2,6}\right)$; ${ }^{13} \mathrm{CNMR}: \quad 109.2\left(\mathrm{CH}-\mathrm{C}_{12}\right), \quad 115.7\left(\mathrm{CH}-\mathrm{C}_{11}\right), \quad 120.7\left(\mathrm{CH}-\mathrm{C}_{13}\right)$, $121.9\left(\mathrm{CH}-\mathrm{C}_{4}\right), 124.3\left(\mathrm{CH}-\mathrm{C}_{3,5}\right), 130.5\left(\mathrm{C}-\mathrm{C}_{10}\right), 139.1\left(\mathrm{C}-\mathrm{C}_{7}\right)$, $142.7\left(\mathrm{C}-\mathrm{C}_{15}\right), 151.2\left(\mathrm{C}-\mathrm{C}_{2,6}\right)$.

\subsection{Antibacterial Activity}

Antibacterial activities of all the prepared heterocyclic compounds were tested via the reported disc-diffusion technique [28], against some bacterial strains, Escherichia Coli, Pseudomonas, Serratia and Bacillus Cereus. A stock concentration of the compounds (3a-g), (100 $\mu \mathrm{g} / \mathrm{mL})$ was made in DMSO. Standard inoculums were distributed evenly, via glass spreader, on the surface of sterilized plates $(9 \mathrm{~cm}$ diameter) containing bacterial medium (nutrient agar broth), and allowed to dry. Sterile discs of $5 \mathrm{~mm}$ diameter were impregnated with each tested compounds $(50 \mu \mathrm{g} / \mathrm{disc})$ using a micropipette and the discs were applied under aseptic technique into the surface of the poisoned agar plates. The plates were incubated for 24 hours at $37^{\circ} \mathrm{C}$. The Inhibition Zone Diameter (IZD) was measured and recorded.

The study was triplicated for each tested compound and the mean was recorded. In absence of photo-irradiation, the synthesized compounds were tested in vitro against the selected bacterial strains. Ampicillin was used as a reference to measure the relative activity under the same conditions.

The study results were reported in Table 1

$\mathrm{IZD}=6-14 \mathrm{~mm}$ beyond control $=+$ (low activity)

$\mathrm{IZD}=15-19 \mathrm{~mm}$ beyond control $=++($ moderate activity $)$.

$\mathrm{IZD}=>19 \mathrm{~mm}$ beyond control $=+++$ (high activity).

\section{Conclusion}

Pyrido[2,3-e]1,3,4-Oxadiazines derivatives were synthesized employing green and sustainable processes through activation with the most renewable source of energy, solar energy, instead the non-green conventional heating. This was accomplished via designing means of parabolic solar reflector and using it in refluxing the reacting mixtures during the reaction course. The green context of the designed benign method was clear enough, introducing, safe, sustainable, low risk, less hazard, time efficient with optimum yield, eco-friendly, and high atom economy practice for preparing valued heterocyclic ring system of recognized application. The antibacterial activity of the resulted compounds was studied and revealed hopeful efficacy, in relation to the referenced antibiotic, against the 
nominated bacterial strains that encouraged further pharmaceutical studies. The article concerned new research window for designing green and sustainable methodologies for constructing new heterocyclic ring systems of considerable biological activity.

\section{References}

[1] Olga, B.; Greta, B.; Rusna, R. and Aurelian, P. G., 2018, DOI: 10.13140/RG.2.2.28236.2624.

[2] Patel, K. D.; Prajapati, S. M.; Panchal, S. N.; and Patel, H. D.; Synth. Comm., 2014, 44(13), 1859-1875.

[3] Abdel-Aziem, A.; Baaiu, B. S.; and Abdelhamid, A. O.; J. Heterocyclic Chem., 2017, doi.org/10.1002/jhet.2970.

[4] Rallas, S.; Gulerman, N.; Erdeniz, H.; and Farmaco, II; 2002, 57, 171-174.

[5] Gursoy, A.; Terzioglu, N.; and Otuk, G.; Eur. J. Med. Chem., 1997, 32, 753-757.

[6] Vicini, P.; Zani, F.; and Cozzini, P.; Eur. J. Med. Chem., 2002, 37, 553-564.

[7] Mamolo, M. G.; Falagiani, V.; Zampieri, D.; Vio, L.; Banfo, E.; and Farmaco, II; 2001, 56, 587-592.

[8] Eissa, Fayez M.; J. Heterocyclic Chem., 2018, 55, 1479.

[9] Rahman, V. M.; Mukhtar, S.; Ansari, W. H.; and Lemiere, G.; Eur. J. Med. Chem., 2005, 40, 173-184.

[10] Dimmock, J. R.; Vashishtha, S. C.; and Stables, J. P.; Eur. J. Med. Chem., 2000, 35, 241-248.

[11] Yapia, R.; La Mara, M. P.; and Massieu, G. H.; Biochem. Pharmacol, 1967, 16, 1211-1218.

[12] Sava, G.; Perissin, L.; Lassiani, L.; and Zabucchi, G.; Chem. Biol. Interact., 1985, 53, 37-43.
[13] Xia, Y.; Chuan-Dong, F.; Zhao, B. X.; Zhao, J.; Shin, D. S.; and Miaom, J. Y.; Eur. J. Med. Chem., 2008, 43, 2347-2353.

[14] Melnyk, P.; Leroux, V.; Serghergert, C.; and Grellier, P.; Bioorg. Med. Chem. Lett., 2006, 16, 31-35.

[15] Ajani, O. O.; Obafemi, C. A.; Nwinyi, O. C.; and Akinpelu, D. A.; Bioorg. Med. Chem., 2010, 18, 214-221.

[16] Zheng, L. W.; Wu, L. L.; Zhao, B. X.; Dong, Y. J.; and Miao, W. L.; Bioorg. Med. Chem., 2009, 17, 1957-1962.

[17] Bhagavan, N. V.; Med. Biochem. 2002, 17, 331-363.

[18] Saulnier, M. G.; Velaprthi, U.; and Zimmermann, K.; Prog In Heterocyclic Synth., 2005, 16, 228-271.

[19] Short, E. I.; Tubercle, 1962, 43, 33-42.

[20] Holdiness, M. R.; Tubercle, 1987, 68, 301-309.

[21] Faroumadi, A.; Kiano, Z.; and Soltani, F.; Open Org. Chem. J., 2010, 4, 1-7.

[22] Mohareb, R. M.; Ibrahim, R. A.; and Moustafa, H. E.; Open Org. Chem. J., 2010, 4, 8-14.

[23] Shindy, H. A., El-Maghraby, M. A.; and Eissa, Fayez. M.; Dyes \& Pig., 2006, 70, 110-116.

[24] Eissa, Fayez M.; and Abdelhameed, R. S.; Green Proc. Synth. 2016, 5, 283-288.

[25] Eissa, Fayez M.; and Abdelghany, A. R.; J. Het. Chem. 2015, DOI: $10.1002 /$ jhet.2428.

[26] Zaidan, MRS.; Rain, AN.; Badrul, AR.; adlin, A.; Norazah, A.; and Zakiah, I.; Trop. Biomed., 2005, 22, 165-170.

[27] Eissa, Fayez M.; J. Chin. Chem. Soc., 2009, 56, 843-849.

[28] Sridhar, SR.; Rajajopal, RV.; Rajavel, R.; Masilamani, S.; and Narasimhan, S.; J. Agric. Food Chem., 2003, 51, 7596-7599. 